\title{
1 Climate influences the response of community functional traits to local conditions
}

2 Laura Melissa Guzman ${ }^{1,2^{*}}$, M. Kurtis Trzcinski3 ${ }^{*}$, Ignacio M. Barberis ${ }^{4}$, Régis Céréghino ${ }^{5}$,

3 Diane S. Srivastava', Benjamin Gilbert ${ }^{6}$, Valério D. Pillar ${ }^{7}$, Paula M. de Omena ${ }^{8,23}$, A.

4 Andrew M. MacDonald ${ }^{5,9}$, Bruno Corbara ${ }^{10}$, Céline Leroy ${ }^{11,12}$, Fabiola Ospina Bautista ${ }^{13}$,

5 Gustavo Q. Romero ${ }^{8}$, Pavel Kratina ${ }^{14}$, Vanderlei J. Debastiani ${ }^{7}$, Ana Z. Gonçalves ${ }^{15}$,

6 Nicholas A.C. Marino ${ }^{16,17}$, Vinicius F. Farjalla ${ }^{16}$, Barbara A. Richardson ${ }^{18,19}$, Michael J.

7 Richardson $^{18,19}$, Olivier Dézerald ${ }^{20}$, Gustavo C.O. Piccoli ${ }^{21}$, Merlijn Jocqué22, Guillermo

8 Montero $^{4}$

9 'Department of Zoology \& Biodiversity Research Centre, University of British Columbia,

10 Vancouver, Canada

11 2Department of Biology, Simon Fraser University, Vancouver, Canada

$12{ }^{3}$ Department of Forest and Conservation Sciences, University of British Columbia,

13 Vancouver, Canada

$14{ }^{4}$ Facultad de Ciencias Agrarias, Instituto de Investigaciones en Ciencias Agrarias de

15 Rosario, Universidad Nacional de Rosario, Consejo Nacional de Investigaciones

16 Científicas y Técnicas, Zavalla, Argentina

17 Laboratoire Ecologie Fonctionnelle et Environnement, Université de Toulouse, CNRS,

18 Toulouse, France

$19{ }^{6}$ Department of Ecology and Evolutionary Biology, University of Toronto, Ontario, Canada

$20{ }^{7}$ Department of Ecology and Graduate Program in Ecology, Universidade Federal do Rio

21 Grande do Sul, Porto Alegre, RS, Brazil

$22{ }^{8}$ Laboratory of Multitrophic Interactions and Biodiversity, Department of Animal Biology,

23 Institute of Biology, University of Campinas, Campinas, SP, Brazil

$24{ }^{9}$ Centre for the Synthesis and Analysis of Biodiversity (CESAB-FRB), Montpellier, France

$25{ }^{10}$ Laboratoire Microorganismes, Génome et Environnement, Université Clermont

26 Auvergne, Aubière, France

$27{ }^{11}$ AMAP, Univ. Montpellier, CIRAD, CNRS, INRA, IRD, Montpellier, France

$28{ }^{12}$ ECOFOG, Campus Agronomique, Kourou, France

$29{ }^{13}$ Departamento de Ciencias Biológicas, Universidad de los Andes, Bogotá, Colombia;

30 Departamento de Ciencias Biológicas, Universidad de Caldas.

$31{ }^{14}$ School of Biological and Chemical Sciences, Queen Mary University of London, London,

32 UK

$33{ }^{15}$ Department of Botany, Biosciences Institute, University of São Paulo, São Paulo, Brazil

$34{ }^{16}$ Departamento de Ecologia, Instituto de Biologia, Universidade Federal do Rio de

35 Janeiro, Rio de Janeiro, RJ, Brazil 
$36{ }^{17}$ Programa de Pós-Graduação em Ecologia, Universidade Federal do Rio de Janeiro, Rio

37 de Janeiro, RJ, Brazil

3818165 Braid Road, Edinburgh, United Kingdom

$39{ }^{19}$ Luquillo LTER, Institute for Tropical Ecosystem Studies, University of Puerto Rico, Rio

40 Piedras, Puerto Rico, USA

$41{ }^{20}$ UMR ESE, Ecology and Ecosystem Health, INRA, Agrocampus Ouest, 35042 Rennes,

42 France

$43{ }^{21}$ Department of Zoology and Botany, University of São Paulo State, São José do Rio

44 Preto, SP, Brazil

$45{ }^{22}$ Aquatic and Terrestrial Ecology, Royal Belgian Institute of Natural Sciences, Brussels,

46 Belgium

$47{ }^{23}$ Institute of Biological Sciences, Universidade Federal do Pará, Belém, PA, Brazil.

48 *Authors contributed equally

49 Correspondence: Laura Melissa Guzman. E-mail: guzman@zoology.ubc.ca

50 Keywords: bromeliads, climatic variation, functional traits, local conditions,

51 macroinvertebrates.

52 Manuscript type: Original research

53 Target Journal: Ecography

\section{Acknowledgements}

55 This research is a product of the FunctionalWebs group funded by the synthesis center

56 CESAB of the French Foundation for Research on Biodiversity (FRB;

57 www.fondationbiodiversite.fr). We acknowledge the support provided by The Natural

58 Sciences and Engineering Research Council of Canada (CGS-D) to L.M.G. and (Discovery

59 Grant) to D.S.S., by UBC Four Year Fellowships to L.M.G., by the Agence Nationale de la 
60 Recherche through an Investissement d'Avenir grant (Labex CEBA,

61 ANR-10-LABX-25-01) to C.L. and R.C., by a BPE-FAPESP grant \#2016/01209-9 to GQR,

62 by CNPq-Brazil research grants to VDP (\#307689/2014-0) and VFF (\#312770/2014-6),

63 by CONICET Proyecto P-UE 22920160100043 and UNR AGR-290 to IMB, and by grants

64 from the Royal Society of Edinburgh, the Carnegie Trust for the Universities of Scotland,

65 the US NSF (DEB-0218039, DEB-0620910), USDA IITF (\#01-1G11120101-001). We

66 acknowledge postdoctoral fellowship support from a PNPD-CAPES grant

67 \#2014/04603-4 to P.M.deO., a PNPD/CAPES grant \#20130877 to N.A.C.M., a CNPq grant

68 (\#401345/2014-9) under the Ciências sem Fronteiras program to V.J.D and a FAPESP

69 grant \#2016/09699-5 to AZG. This is a publication of the Bromeliad Working Group. 
bioRxiv preprint doi: https://doi.org/10.1101/2020.10.30.362525; this version posted November 1,2020 . The copyright holder for this preprint (which was not certified by peer review) is the author/funder, who has granted bioRxiv a license to display the preprint in perpetuity. It is made available under aCC-BY 4.0 International license.

70 Climate influences the response of community functional traits to local conditions

71 Keywords: bromeliads, climatic variation, functional traits, local conditions,

72 macroinvertebrates.

73 Manuscript type: Original research

74 Target Journal: Ecography 


\section{Abstract}

76 Functional traits determine an organism's performance in a given environment and as

77 such determine which organisms will be found where. Species respond to local

78 conditions, but also to larger scale gradients, such as climate. Trait ecology links these

79 responses of species to community composition and species distributions. Yet, we often

80 do not know which environmental gradients are most important in determining

81 community trait composition at either local or biogeographical scales, or their interaction.

82 Here we quantify the relative contribution of local and climatic conditions to the structure

83 and composition of functional traits found within bromeliad invertebrate communities. We

84 conclude that climate explains more variation in invertebrate trait composition within

85 bromeliads than does local conditions. Importantly, climate mediated the response of

86 traits to local conditions; for example, invertebrates with benthic life-history traits

87 increased with bromeliad water volume only under certain precipitation regimes. Our

88 ability to detect this and other patterns hinged on the compilation of multiple fine-grained

89 datasets, allowing us to contrast the effect of climate vs. local conditions. We suggest

90 that, in addition to sampling communities at local scales, we need to aggregate studies

91 that span large ranges in climate variation in order to fully understand trait filtering at

92 local, regional and global scales. 


\section{Introduction}

94 Ecologists are reformulating long-held perspectives on biodiversity using functional traits.

95 Since organisms interact with their environment through their traits, patterns in species

96 distribution should be a direct function of their traits. Traits directly affect community

97 assembly and species interactions, such that any snapshot of a community is the result of

98 i) physiological tolerances (Winemiller et al. 2015, Pianka et al. 2017), ii) species

99 interactions (Chesson 2000, Chase and Leibold 2004, Estes et al. 2011), iii) dispersal and

100 priority effects (MacArthur and Wilson 1967, Diamond 1975, Hanski 1999), iv) demographic

101 stochasticity (Lande et al. 2003, Hubbell 2011), and v) phylogenetic constraints (Vellend

102 2016, Pianka et al. 2017). Most of these mechanisms are, to some degree, driven by

103 functional traits (mechanisms i-iii) or determine the distribution of traits (mechanism v),

104 and their prevalence and diversity modulate the relationship between biodiversity and

105 ecosystem function rather than species per se (Schmitz et al. 2015). Similarly, the

106 redundancy of functional traits in a community can maintain ecosystem function and

107 stability in the face of environmental change and species loss (Loreau et al. 2001). By

108 shifting our focus away from taxonomic diversity to the diversity of functional traits within

109 a community or metacommunity, we strengthen our ability to detect the mechanisms that

110 underlie observed patterns in species distribution and biodiversity (McGill et al. 2006). In

111 particular, functional traits have been associated with broad biogeographic patterns, such

112 as the latitudinal gradient in biodiversity, leading to new insights into the processes and

113 causes of global biodiversity (Chave et al. 2009, Ricklefs 2012, Lamanna et al. 2014). 
114 This trait-based paradigm recasts community ecology's central question about species

115 diversity and coexistence as: which processes determine the functional trait composition

116 of ecological communities? Spatial scale is implicit in this question, as different processes

117 are expected to act at different scales (Levin 1992, Chave 2013). For example, species

118 interactions are expected to be strongest at small spatial scales, whereas environmental

119 filtering often occurs at spatial scales large enough for environmental gradients to

120 exceed physiological thresholds (Kraft and Ackerly 2010). Finally, processes like dispersal

121 limitation and biogeographical constraints to the species pool often operate at the

122 largest spatial scales (Ricklefs and Schluter 1993). The grand challenge of integrating

123 these multiple scale processes on traits can only be resolved by a synthesis of traditional

124 small-scale ecology with macroecology and biogeography (Violle et al. 2014).

125 Environmental conditions could have cross-scale effects on functional traits in several

126 ways. First, local factors (e.g. resource availability) could scale up to affect the geographic

127 distribution of functional traits, or, local conditions such as microclimate may compensate

128 apparently limiting climatic conditions. Second, climatic and biogeographic factors could

129 constrain the local distribution of functional traits and thereby impact local processes. A

130 practical difficulty of including large scale environmental factors or biogeography into

131 syntheses of local scale studies is that some variables will be spatially pseudo-replicated

132 and others will not. That is, several field studies that fall within a single climatic zone or

133 geographic region may not represent independent measures, yet a large number of

134 sampling units are needed to detect local effects on community functional trait 
135 composition. These issues can be partially resolved by a spatially structured hierarchical

136 analysis.

137 The theory and motivation for our study could be applied to almost any ecological

138 community. Yet, the data required to test this theory requires extensive information on

139 species composition, functional traits, local conditions and climate for multiple

140 georeferenced occurrences of a defined community across a broad geographic range;

141 such data are rarely available for multitrophic communities. Here we use the aquatic

142 macroinvertebrates in tank bromeliads as a model system to understand community

143 structure. The invertebrate communities in tank bromeliads have proven to be useful

144 systems for testing ecological theory, as they are easily manipulated and censused and

145 are naturally highly replicated. Previous work has related ecosystem functions, trophic

146 structure and system resilience to a variety of invertebrate functional traits (Dézerald et

147 al. 2015, 2018, de Omena et al. 2019). To date, researchers of this system have primarily

148 focused on local-scale explanations for community composition and ecosystem function,

149 detailing how bromeliad size (volume of aquatic habitat), detrital inputs, and canopy

150 cover affect community composition (Petermann et al. 2015, Kratina et al. 2017). However,

151 much of the geographic variation in invertebrate composition remains unexplained, and

152 consequently begs for more explicit incorporation of broad-scale variables like climate.

153 For example, extreme rainfall events lead to an inversion of the trophic pyramid of

154 macroinvertebrates in bromeliads across seven study sites broadly distributed across the

155 neotropics (Romero et al. 2020). 
156 Here, we used fine-grain data on the functional traits of aquatic macroinvertebrates

157 sampled in more than 1600 tank bromeliads across 18 climate zones throughout the

158 Neotropics to partition the separate and combined effects of local conditions and climate

159 on community trait composition. Based on previous studies of bromeliad

160 macroinvertebrate ecosystems, we expect that both local conditions and climatic

161 gradients affect trait composition (Céréghino et al. 2011, 2018, Dézerald et al. 2015). We

162 tested three hypotheses of how trait variation could be partitioned between local and

163 biogeographical environmental gradients: (i) Variation in trait composition is primarily

164 explained by variation in the environment at the biogeographic scale. This would occur

165 when climatic factors, which vary only at biogeographic scales, determine the cost and

166 benefits of different functional traits. For example, temperature determines development

167 rates (Damos and Savopoulou-Soultani 2012) and predation pressure (Romero et al.

168 2018), while precipitation determines the mortality rates due to desiccation (Amundrud

169 and Srivastava 2015). (ii) Variation in trait composition occurs primarily along regional

170 environmental gradients, or the interaction between local conditions and the climate at a

171 given region. This would occur when heterogeneity in local conditions determines life

172 history traits. For example, traits may vary as a response to the availability of resources

173 within a bromeliad (Srivastava et al. 2008) and the avoidance of negative species

174 interactions (Hammill et al. 2015). (iii) Neither local nor regional conditions determine trait

175 composition. This would occur at the regional scale if strong biogeographic constraints

176 dampen any effect climate may have on traits. For example, geographic dispersal

177 barriers on particular clades (Amundrud et al. 2018), coupled with deep phylogenetic 
178 signal on some traits override the effects of climatic conditions. Similarly, at a local scale,

179 stochasticity in colonization coupled with noise added by a generalist predator can

180 overwhelm any signal caused by environmental gradients (Srivastava and Bell 2009). We

181 used hierarchical analyses to distinguish between these three hypotheses by testing the

182 effects of local conditions, climate, and their interaction on the community functional

183 traits of bromeliad invertebrates at local and bioclimatic scales.

\section{Methods}

\section{Invertebrate traits}

186 We used as functional trait values the species scores on four main axes of invertebrate

187 trait variation identified by Cereghino et al. (2018), which represent life-history strategies

188 along trophic, habitat, defence and life-history niche axes describing ca. 852 aquatic

189 invertebrate taxa occurring in Neotropical tank bromeliads, mostly identified to species

190 or morphospecies (hereafter "species"). For completeness, in the following we explain

191 the method used by Cereghino et al. (2018) to identify such a synthetic traits: Each of

192 these species was characterized by 12 nominal traits: maximum body size, aquatic

193 developmental stage, reproduction mode, dispersal mode, resistance forms, respiration

194 mode, locomotion mode, food, feeding group, cohort production interval, morphological

195 defence, and body form. Each nominal trait had a number of modalities, or states. For

196 example, the states for the trait "feeding group" were "deposit feeder", "shredder",

197 "scraper", "filter-feeder", "piercer" and "predator". A full description of traits and states

198 can be found in Céréghino et al. (2018). Information on these traits was structured using a 
199 fuzzy-coding technique (Chevenet et al. 1994): scores ranged from "O” indicating "no

200 affinity", to "3" indicating "high affinity" of the species for a given trait state. Scores were

201 based on observations of specimens (Dézerald et al. 2017), on the scientific literature

202 (e.g. Merritt and Cummins 1996, Céréghino et al. 2011) and expert opinion (see Céréghino

203 et al. 2018 for list of traits, modalities, and their definitions) the species $x$ trait matrix is

204 available at https://doi.org/10.5281/ zenodo.1200194. Principal Component Analysis (PCA)

205 was used to reduce trait dimensionality to significant axes of trait variation. The

206 rank-transformed [species $x$ trait] matrix was used to compute Spearman's rank

207 correlations between trait modalities, which then underwent PCA with bootstrap

208 resampling (Pillar, 1999). This procedure allowed us to test ordination stability and to

209 interpret the significant ordination axes in light of correlations with trait states. We

210 identified a robust set of four orthogonal and important axes of trait variation, namely

211 trophic position, habitat use, morphological defence, and life cycle complexity (Céréghino

212 et al. 2018). The species scores for these four PCA axes (available at

213 https://doi.org/10.5281/zenodo.1200194) thus represented continuous trait values, or

214 synthetic traits, which we then used in analyses of the processes underlying functional

215 diversity across different spatial scales in relation to environmental factors (this study).

216 Site sampling

217 The data for the present study consists of the abundance of macroinvertebrates found

218 within 1436 bromeliads in 18 field sites in 8 countries (Bromeliad Working Group BWG

219 database, Table A1). While the Bromeliad Working Group has sampled more countries 
220 and field sites, we only use the sites where more than 15 bromeliads were sampled.

221 Some of these field sites were visited in multiple years (Table A1), while others were

222 visited only once. While we acknowledge there are temporal trends in community

223 abundance and composition, all of our sampling units (each sampled bromeliad) are a

224 snap-shot of the community structure, so we made the simplifying assumption to treat all

225 sampling units within a site the same, regardless of the year in which they were

226 collected. Examining temporal trends in trait composition, would be an interesting follow

227 up study.

228 The full suite of 19 bioclimatic variables was extracted from WorldClim using the latitude

229 and longitude of each sampled site (Fick and Hijmans 2017). Some of our sampling

230 locations were within $1 \mathrm{~km}^{2}$ of one another. As such, these locations had the same

231 climatic conditions since this is the smallest resolution in WorldClim. We decided to group

232 the data collected from these locations, instead of attempting to downscale the climatic

233 conditions to a smaller resolution. After grouping the data from these locations, we

234 ended up with 18 sites, which we refer to as bioclimatic zones.

\section{Bromeliad sampling}

236 The following bromeliad genera were sampled across all sites: Neoregelia, Quesnelia,

237 Tillandsia, Guzmania, Vriesea, Aechmea and Catopsis. Each tank bromeliad was

238 exhaustively sampled either by dissecting the rosette, or by pipetting out all of its

239 contents. The bromeliad macroinvertebrate community is defined as all the aquatic 
240 invertebrates found by the naked eye $(>0.5 \mathrm{~mm})$ within a single plant. A total of 637

241 morpho-species were found.

242 Three local conditions were collected for each plant: water volume at time of sampling

$243(\mathrm{~mL})$, total amount of detritus (mg), and canopy cover (either open or closed canopy). The

244 data on total amount of detritus and the water volume at the time of sampling were

245 log-transformed before the analyses. These variables are proxies of habitat size and

246 energy inputs, which are key drivers of food web structure (Oertli et al. 2002, Moore et

247 al. 2004). The total amount of detritus was calculated by adding the amount of small,

248 medium and large detritus. In $25.8 \%$ of bromeliads, total detritus was not measured

249 directly, instead we imputed total detritus with allometric relationships using other size

250 categories of detritus, and in a few cases with the number of leaves and the diameter of

251 the plant. In $50.4 \%$ of bromeliads, total water volume was not measured directly and

252 instead we imputed total water volume using leaf size, plant species, plant height, plant

253 diameter, and number of leaves. When either of these variables were missing, we used

254 generalized linear models with a Gaussian error distribution to impute missing values.

255 Thus, the dataset was a combination of directly measured and estimated values.

\section{Spatial scale of environmental variation}

257 To better understand the spatial scale of environmental variation, we partitioned the

258 variation in local environmental conditions of a bromeliad into site and bioclimatic scales

259 using three nested hierarchical models, one for each response. All three models used 
260 the same structure of random effects, but different likelihoods according to the

261 environmental variable. The environmental variables partitioned in this way included log

262 detritus (normal likelihood) log water volume (normal) and canopy cover (binomial). By

263 using nested random effects, we can partition the variation of each local environmental

264 condition by spatial scale and determine at which spatial scale most of the variation is

265 explained. For each environmental variable, we estimated random effects for the field

266 visit (site by year combination) within bioclimatic zone, and bioclimatic zone. We also

267 calculated the correlation between all local conditions both within and across all

268 bioclimatic zones.

269 Trait analysis

270 Since our unit of analysis is the bromeliad invertebrate community, it was necessary to

271 quantify the presence and abundance of the animals and their traits for each bromeliad.

272 To do this, we calculated the community weighted means (CWM) of each synthetic trait

273 for each bromeliad (local scale analysis). Community weighted means (CWM) was given

274 by:

275

$C W M=\sum_{i=1}^{n} p_{i} x_{i}$

276 where $p_{i}$ is the proportion of species abundance, $x_{i}$ is the trait value of that species and $n$

277 is the number of species in that community (Garnier et al. 2004, Swenson 2014). To

278 reduce the effect of highly abundant species on the analysis, we applied a square root 
279 transformation of the proportional abundance of invertebrates within a bromeliad (i.e. a

280 Hellinger transformation). This transformation was necessary since we used abundance

281 and not biomass, and the most abundant species were orders of magnitude (up to 100s

282 of individuals) more abundant than the least abundant species (1 or 2 individuals)

283 (Legendre and Gallagher 2001). The square root transformation de-emphasizes

284 superabundant species (gave a more equal weight to rare species), and the community

285 weighted means (CWM) allowed us to characterize the relative abundance of traits in the

286 sampled bromeliad.

287 Community weighted means were obtained using the data version 0.7.7 extracted from

288 the BWG database in July 2017.

289 To determine the effects of environmental conditions on the CWM computed using the

290 species scores on the four synthetic traits, we used a permutational multivariate analysis

291 of variance - PERMANOVA (vegan R package; Anderson 2001). This method is based on

292 within- and between-group sums of squares computed on pairwise dissimilarities, in this

293 case, of bromeliad communities considering the CWM trait values. However, instead of

294 permuting the site matrix (bromeliads or bioclimatic zones), we adapted the method to

295 permute among the species vectors in the trait matrix and recomputed the CWMs, to

296 reduce the risk of type I error (Peres-Neto and Kembel 2015, Hawkins et al. 2017, Zelený

297 2018). Since each bioclimatic zone differs in the number of bromeliad communities

298 sampled (Table A1), and sample size may bias the relative amount of variance explained,

299 we devised a sub-sampling scheme where we randomly selected 15 bromeliads from a 
300 randomly selected field visit within a bioclimatic zone (18 zones, Figure 1). Note that the

301 minimum number of bromeliads per site is 18 (Table A1), therefore for some sites, most

302 bromeliads are selected in every sub-sampling procedure. We found that 15 bromeliads

303 is the minimum number that still provides a comprehensive sample of the community

304 within a field visit. Every time we performed this sub-sampling procedure, we ran the

305 multivariate statistical analysis and compiled the main results (sum of squares). We

306 repeated this process 1000 times. We used the marginal sum of squares in the analysis

307 without interactions and the sequential sum of squares in the analysis with interactions to

308 the variation explained by the main effects. From these runs, we obtained a distribution

309 of P-values and sums of squares. We do not report P-values of individual runs, because

310 they do not represent valid independents tests, however, we do report the P-values of a

311 non-sub-sampled analysis in the appendix (Tables A3 and A4). Some of the distributions

312 of sums of squares were skewed, while some were normally distributed (Figure A5). To

313 summarize this variation, we first calculated the mean of the sum of squares explained by

314 each predictor across all sub-samplings, and then calculated the total contribution of

315 local conditions, climate variables, and their interaction. This procedure allowed us to

316 take advantage of the central limit theorem to ensure that the addition occurs on

317 normally distributed means.

318 We performed the multivariate analysis of variance for each spatial scale to replicate a

319 'fine-grained analysis' (Figure 1a), a 'coarse-grained analysis' (Figure 1b) and a

320 fine-grained analysis with resource-climate interactions (Figure 1c). Thus, we are able to 

compare the explanatory power of these models to explain functional trait composition if

322 we only had local conditions, climate information or both. (i) For the fine-grained analysis, 323 we used each bromeliad as the sampling unit and only tested the effects of local

324 conditions (that is the environmental conditions that were measured for every bromeliad:

325 water level, detritus amount, and canopy cover). We restricted the sub-sampling to field

326 visits (site by year combination) to ensure that macroinvertebrate traits relating to species

327 that only occurred in a single bioclimatic zone (most species) were not mixed between

328 countries or years (Figure 1 - fine-grained analysis). Analysis using bromeliads as

329 sampling units and only climatic variables as predictors were used to filter the 19

330 bioclimatic variables to a smaller subset. We retained climatic variables that explained a

331 significant proportion of variation in at least $5 \%$ of the runs (BC2, 4, 15 and 17), and which

332 were then used in subsequent analyses. In 1000 randomizations we expect at least $5 \%$ of

333 runs to appear significant by chance (type I error), so we only report explanatory

334 variables that are significant in $>5 \%$ of runs. (ii) For the coarse-grained analysis, we used

335 the 'bioclimatic zone' as units for which we calculated the species pool CWM by

336 summing the abundance of all the morphospecies across the sub-sampled 15 bromeliads

337 and only tested the effects of climatic variables (Figure 1 - coarse-grained analysis). (iii) To

338 test for the interactions between climate and local conditions, we used the bromeliad as

339 the sampling unit, and tested the effect of local conditions, climate and their interactions

340 (Figure 1 - fine-grained analysis with resource-climate interactions). We did not include

341 the interaction between canopy cover and climatic conditions because few bioclimatic 
343 confounded with bioclimatic zone.

344 All multivariate analyses were performed using the vegan package (Oksanen et al. 2017).

345 Mixed effect models were performed using Ime4 R package (Bates et al. 2015) and all

346 analyses were done using the $\mathrm{R}$ programming language (R Core Team 2016). The code

347 for the sub-sampling and statistical analysis, as well as the adaptation of the

348 PERMANOVA can be found in: https://github.com/Imguzman/Climate invertebrate traits.

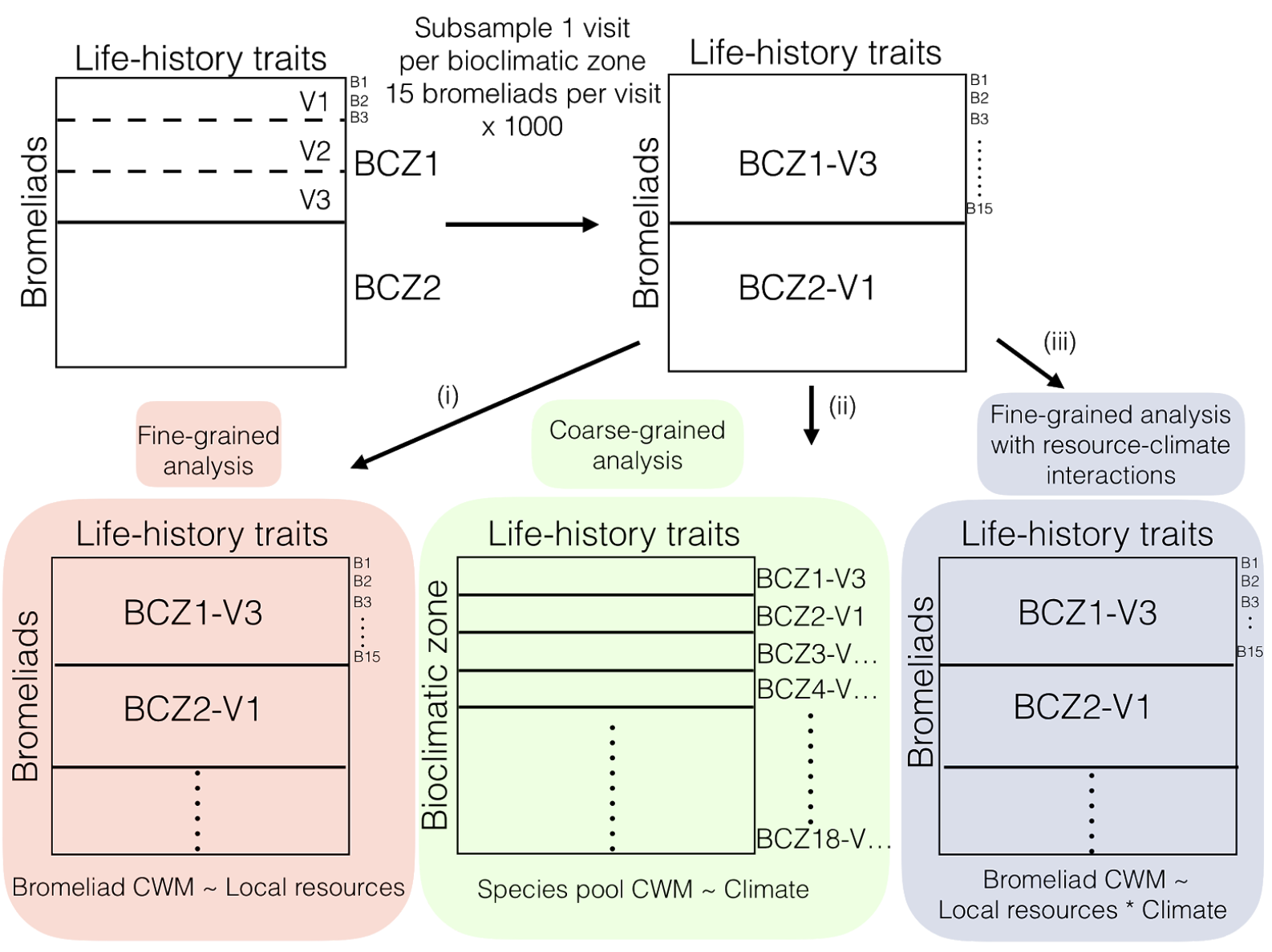


349 Figure 1: Schematic representation of the analysis. We tested for the effect of environmental conditions on

350 trait composition in three main analyses. BCZ1, BCZ2, BCZ... represent the bioclimatic zone, which can

351 contain multiple visits V1, V2, V3, V... We sampled one visit for each bioclimatic zone represented by

352 BCZ1-V3, that is, bioclimatic zone 1, visit 3. (i) At the fine grained (red area) analysis, we tested for the effect

353 of local conditions on bromeliad weighted means (CWM). (ii) At the coarse-grained analysis (green area),

354 we tested for the effect of climate on species pools CWM. (iii) We tested the interaction between climate

355 and local conditions in determining bromeliad CWM (blue-gray area).

\section{Results}

\section{Spatial scale of environmental variation}

358 We determined the spatial scale of variation in our three local conditions: total detritus,

359 water volume at time of sampling, and whether the canopy was open or closed. This

360 analysis gave some indication of the potential power of each variable to explain variation

361 in synthetic trait composition (i.e. little variation at a given scale indicates a lower

362 likelihood of a significant effect at that given scale). Variation in total detritus was greatest

363 at the level of the bioclimatic zone (39.2\% of variation) but also was high at the local scale

364 of the field site (31\%). Variation in water volume was greatest at the local scale of field

365 visits (42.6\%) and minimal at the level of bioclimatic zone (2.7\%). Finally, variation in

366 canopy cover was highest at the level of bioclimatic zone (73.5\%) and lowest at the level

367 of field visit (26\%) (Figure A3). We also found that these three local conditions were only

368 weakly correlated across and within zones. The correlation values across bioclimatic

369 zones ranged between -0.01 and 0.35 , while the correlation values within bioclimatic

370 zones ranged between -0.32 and 0.28 (Table A2). 
371 Fine-grained analysis

372 Invertebrate traits varied among every bromeliad within a field visit (Figure 2). We found

373 that only a small amount of trait variation in CWMs (6.1\%) could be explained by local

374 conditions, and that no single local condition explained most of this variation. The

375 amount of variation explained ranged from zero to $19.7 \%$ depending on the subset of

376 bromeliads selected, and the distribution of variation explained was skewed (Figure A1,

377 Table 1 - Fine-grained analysis). 


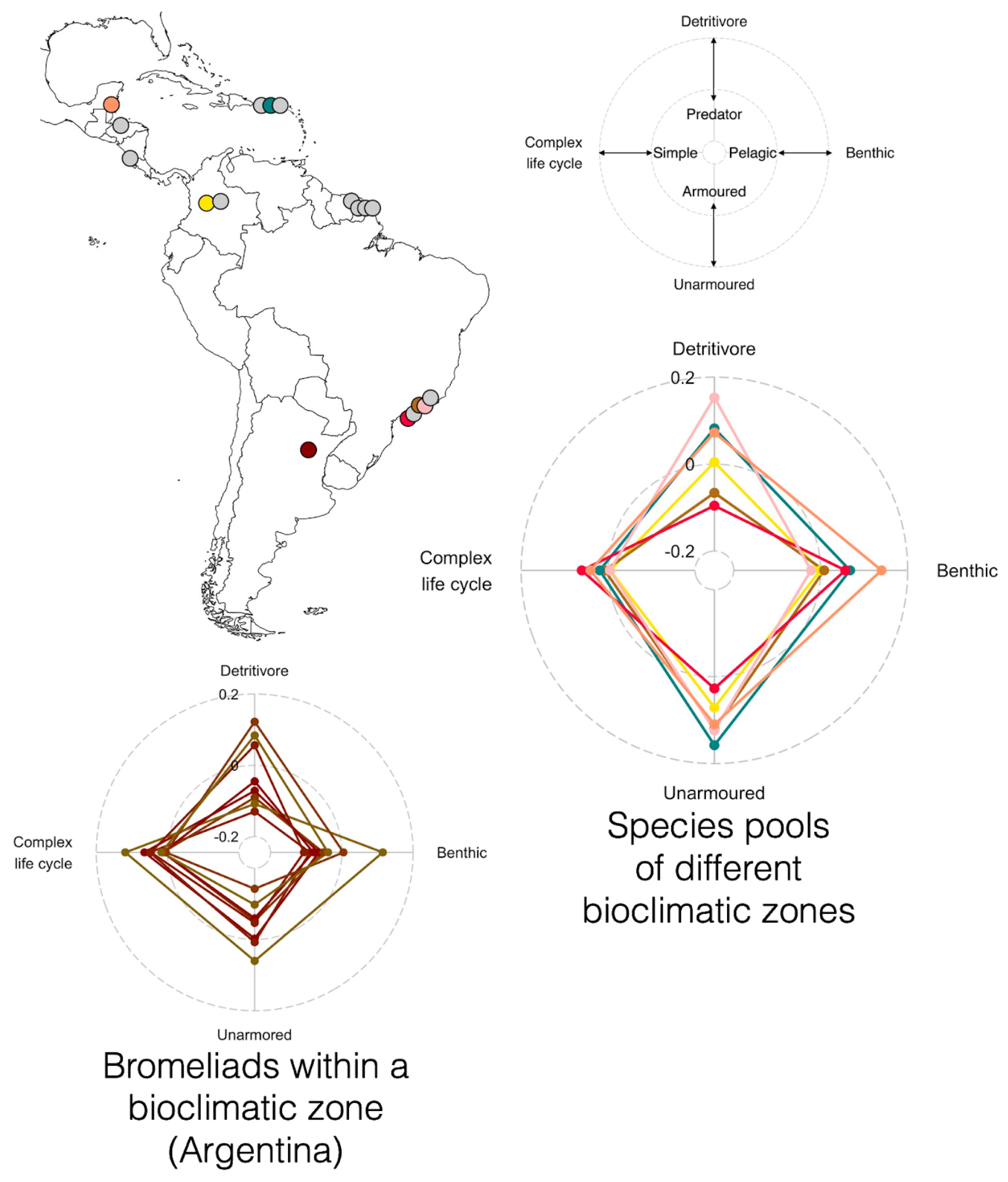

378 Figure 2: Invertebrate traits at the level of bromeliads and at the level of species pools, for bromeliads

379 sampled in bioclimatic zones throughout the Neotropics (map, top left). Empty spider plot (top right) shows

380 all the four trait axes and their directions, and forms a key for the two filled spider plots (bottom). Filled 
bioRxiv preprint doi: https://doi.org/10.1101/2020.10.30.362525; this version posted November 1,2020 . The copyright holder for this preprint (which was not certified by peer review) is the author/funder, who has granted bioRxiv a license to display the preprint in perpetuity. It is made available under aCC-BY 4.0 International license.

spider plots summarize the CWM of the four trait axes in a single bromeliad (bottom left) and the CWM in some example species pools (bottom right). Colours on spider plots correspond to bioclimatic zones on map, with those zones not shown in spider plots indicated in grey on the map.

384 Table 1. Synthetic trait composition (CWM) explained by local conditions in the fine-grained analysis and by climatic variables in the coarse-grained analysis. The analysis using the local conditions uses the CWM for each bromeliad. This analysis is blocked within each bioclimatic zone. The analysis using the biogeographic climatic variables uses the CWM for the species pool for each bioclimatic zone.

\begin{tabular}{|l|l|l|}
\hline \multirow{2}{*}{ Predictor } & \multicolumn{2}{|c|}{ Percentage of total sum of squares } \\
\cline { 2 - 3 } & Mean & Median \\
\hline Canopy cover & 2.68 & 2.19 \\
\hline Detritus & 2.30 & 1.92 \\
\hline Water volume & 1.18 & 0.99 \\
\hline Full model & 6.16 & 5.1 \\
\hline & \multicolumn{1}{|c|}{ Coarse-grained analysis } \\
\hline $\begin{array}{l}\text { Mean Diurnal Temperature } \\
\text { Range }\end{array}$ & 14.7 & 14.7 \\
\hline Temperature Seasonality & 10.2 & 10.1 \\
\hline $\begin{array}{l}\text { Precipitation of Driest } \\
\text { Quarter }\end{array}$ & 7.64 & 7.69 \\
\hline Precipitation Seasonality & 7.39 & 7.25 \\
\hline Full model & 39.93 & 39.44 \\
\hline
\end{tabular}


389 Species pools differed in the relative proportions of invertebrate traits (Figure 2). In the

390 coarse-grained analysis we found that climatic variables explained on average $39.9 \%$ of

391 the variation in the trait composition of species pools (Table 1 - Coarse-grained analysis).

392 The range of explained variation was large (14 to 47\%) depending on the subset of

393 bromeliads selected (Figure A2). The amount of variation explained in this analysis is not

394 necessarily directly comparable to that in the fine-grained analysis because the scale of

395 the response variable (CWMs) is different; for this analysis, we aggregated species at the

396 site rather than bromeliad level. This aggregation changes the mean by weighting

397 bromeliads with more individuals more heavily, and also reduces the number of

398 observations, which tends to raise the R2 values. Four out of 19 bioclimatic variables

399 explained substantial variation in trait composition of the macroinvertebrates across the

400 Neotropics: mean diurnal range in temperature (BC2), temperature annual seasonality

401 (BC4), precipitation annual seasonality (BC15), and precipitation of the driest quarter

402 (BC17) (Figure A4; Table 1). Species pools from zones with high mean diurnal temperature

403 range and high precipitation seasonality tended to be dominated by armoured

404 invertebrates (Figure A5d, A7d). These climatic variables also differed in their effect on

405 trophic traits: detritivores were favoured in zones with high precipitation in the driest

406 quarter (Figure A8c), whereas predators were favoured in zones with high precipitation

407 seasonality and mean diurnal range (Figures A5c, A7c).

408 Fine-grained analysis with resource-climate interactions 
409 The full model - using both the climatic and local resource environmental gradients to

410 explain traits within individual bromeliads - explained between 27.2 and $44.1 \%$ of trait

411 variation, when all the explanatory variables were included, with an average of $36.5 \%$ of

412 the variation explained (Figure A9). We found that the local conditions explained 8.7\%,

413 climate explained $17.7 \%$, and their interaction explained $10 \%$ of the variation in community

414 weighted functional traits (CWMs) on average across all runs. Among the local conditions

415 tested, detritus explained more variation than canopy cover or water volume. Of the

416 climatic conditions tested, mean diurnal range in temperature (BC2) explained more

417 variation than did the other climatic variables. Bromeliads with high mean diurnal range in

418 temperature typically had more complex and unarmoured invertebrates (Figure A10). The

419 cross-scale interaction that explained the most variation was detritus amount and

420 temperature seasonality (BC4; Figure 3-4, Figures A11, Table 2). Specifically, detritus-rich

421 bromeliads in zones with seasonal temperatures tended to contain more unarmoured

422 invertebrates, predators, and invertebrates with complex life cycles (Figure 4). No single

423 explanatory variable consistently explained the most variation in CWMs, rather, each

424 variable contributed a small amount to the total amount of variation explained by the full

425 model, which taken together explained more than a third of the variation in functional

426 traits.

427 Table 2: Synthetic trait composition explained by local conditions, biogeographic climatic variables and

428 their interactions. This analysis used the CWM for each bromeliad. We did not include the interaction

429 between canopy cover and climatic conditions because few bioclimatic zones had both open and closed

430 canopy, therefore canopy cover would be confounded with bioclimatic zone.

\begin{tabular}{|l|l|l|}
\hline \multirow{2}{*}{} & \multicolumn{2}{|l|}{ Percentage of total sum of squares } \\
\cline { 2 - 3 } & Mean & Median \\
\hline & Local conditions \\
\hline
\end{tabular}




\begin{tabular}{|l|l|l|}
\hline Canopy cover & 2.43 & 1.86 \\
\hline Detritus & 4.95 & 4.86 \\
\hline Water volume Climatic variables & 1.35 & 1.09 \\
\hline \multicolumn{2}{|c|}{} \\
\hline Mean Diurnal Temperature Range & 5.55 & 5.55 \\
\hline Temperature Seasonality & 3.55 & 3.31 \\
\hline Precipitation of Driest Quarter & 4.66 & 4.57 \\
\hline Precipitation Seasonality & 3.97 & 3.99 \\
\hline \multicolumn{1}{|c|}{ Interactions between local conditions and climatic variables } \\
\hline $\begin{array}{l}\text { Detritus : Mean Diurnal Temperature } \\
\text { Range }\end{array}$ & 1.23 & 1.0 \\
\hline Detritus : Temperature Seasonality & 2.22 & 2.12 \\
\hline Detritus : Precipitation of Driest Quarter & 1.33 & 1.25 \\
\hline Detritus : Precipitation Seasonality & 1.56 & 1.39 \\
\hline $\begin{array}{l}\text { Water volume: Mean Diurnal } \\
\text { Temperature Range }\end{array}$ & 0.84 & 0.68 \\
\hline $\begin{array}{l}\text { Water volume: } \\
\text { Temperature Seasonality }\end{array}$ & 36.49 & 36.62 \\
\hline $\begin{array}{l}\text { Water volume: } \\
\text { Precipitation of Driest Quarter }\end{array}$ & 1.37 & 1.20 \\
\hline Water volume: Precipitation Seasonality & 0.52 & 0.44 \\
\hline Full model & 0.85 \\
\hline
\end{tabular}


Low detritus

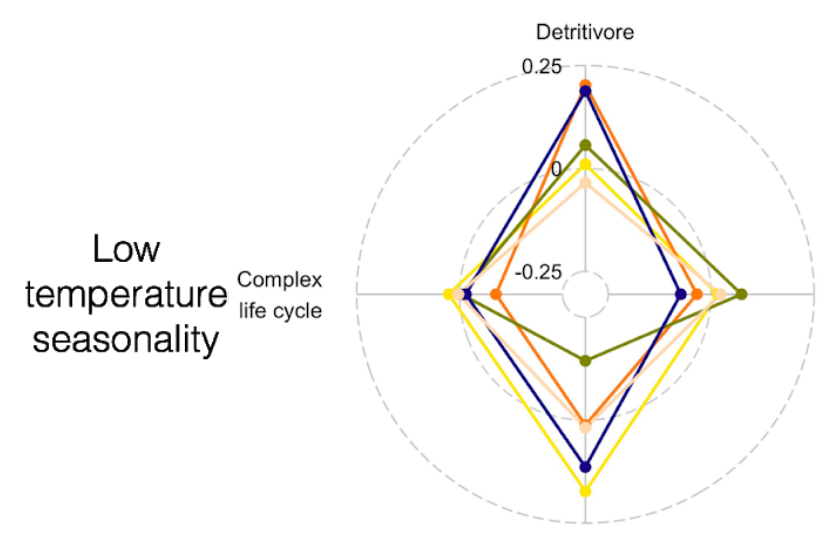

Unarmoured

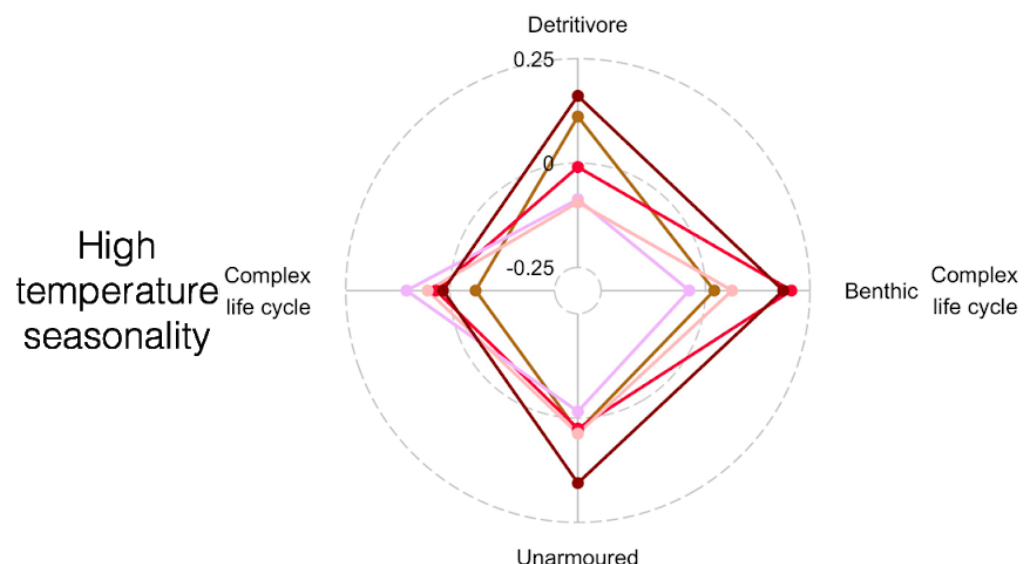

Unarmoured
High detritus
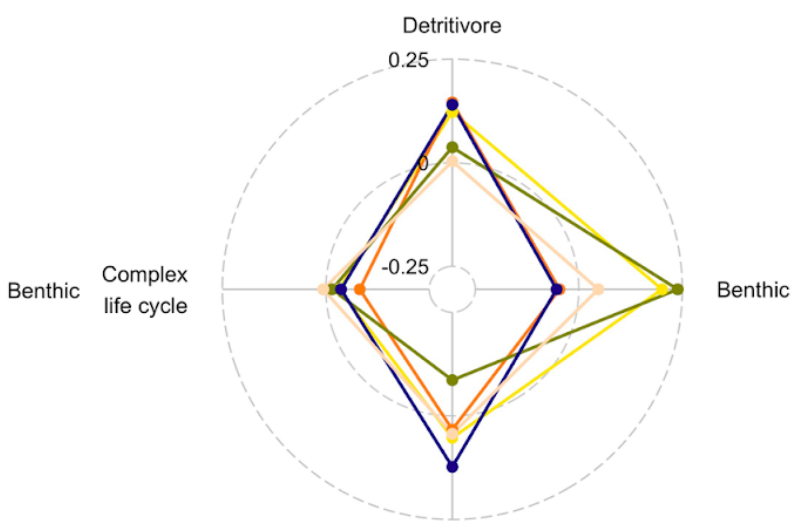

Unarmoured

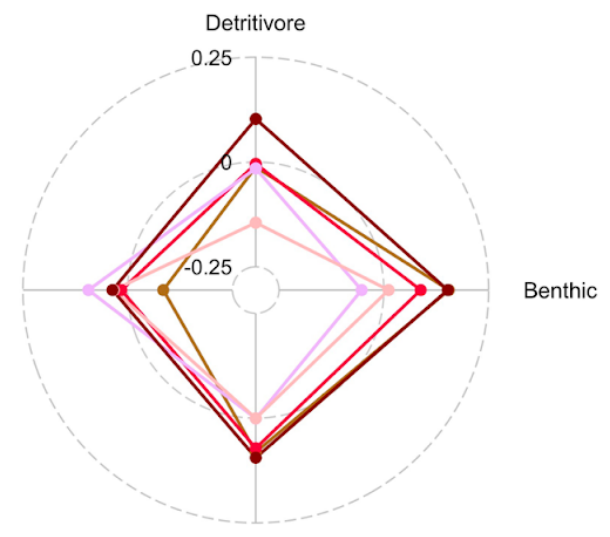

Unarmoured

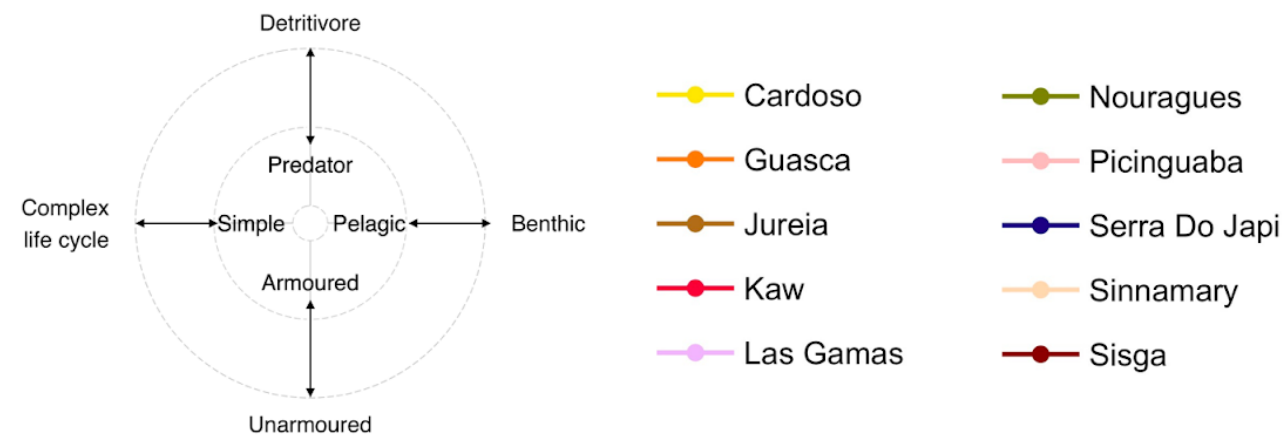

431 Figure 3: Bromeliads are characterized by their community-weighted mean traits for bioclimatic zones with

432 low and high detritus, and high and low temperature seasonality. Detritus affects particularly the 
433 Benthic-Pelagic axis: bromeliads with high detritus have more benthic organisms (i.e. high Benthic-Pelagic

434 axis values). Bioclimatic zones with high temperature seasonality have more organisms with complex life

435 cycles (i.e. higher values in the Complex life cycle - Simple axis).

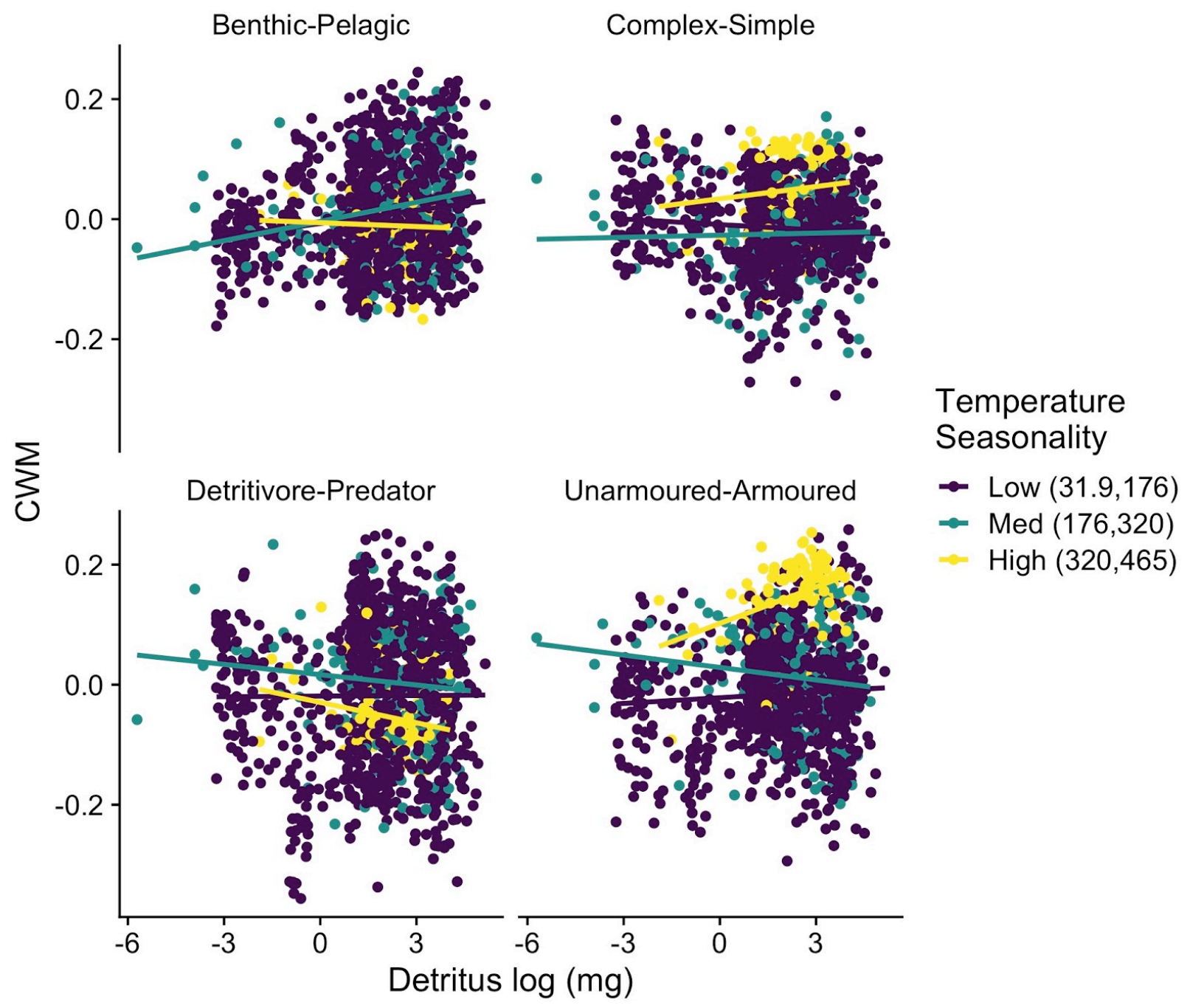

436 Figure 4: The four trait axes differ in their relationship with the total amount of detritus which is also

437 mediated by temperature seasonality. Each point is the community weighted mean of a single bromeliad.

438 The coloured lines are simple linear regressions intended only to improve the visualization of the data and

439 not meant to be used for formal analysis since the CWM are multivariate. 
441 Our analyses demonstrate the importance of climate and cross-scale interactions of

442 climate with local conditions on the functional traits of macroinvertebrate communities.

443 Climate explained $40 \%$ of the trait variation in regional species pools, corroborating the

444 hypothesis that trait composition is primarily explained by the environment at the

445 biogeographic scale. Climate also explained a substantial amount (28\%) of trait variation

446 at the scale of individual bromeliads, both directly (18\%), and by modifying the response

447 of traits to local conditions in cross-scale interactions (10\%) (Figure A9). By contrast, local

448 conditions of bromeliads, by themselves, explained only 6-9\% of trait variation depending

449 on the model used. The fact that we were able to explain trait variation with both climate

450 and local conditions means that functional traits are not entirely determined by stochastic

451 factors, historical contingency and phylogenetic constraints. Although stochastic and

452 biogeographic factors no doubt affect trait distribution, they are not strong enough to

453 completely overwhelm or erase the effects that climate and local conditions have on the

454 functional traits of bromeliad macroinvertebrate communities. The implication is that local

455 adaptation and/or filtering occurs in response to both local and climatic conditions, and

456 ultimately shapes the ecological communities of bromeliad macroinvertebrates and their

457 suite of traits.

458 The most important climatic factor in explaining trait variation was mean diurnal range of

459 temperature. Combinations of precipitation and temperature were also important. 
460 Detritus and canopy cover were the most important local conditions, but only explained

$4616.1 \%$ of the variation in invertebrate trait composition. In general, not a single predictor

462 (either local conditions or climate) explained very large amounts of variation in trait

463 composition. Instead, the aggregated effects of each predictor contributed to the total

464 variation explained.

465 Our fine-grained analysis with cross-scale interactions allowed us to conclude that

466 climate explains more variation in macroinvertebrate trait composition than local

467 conditions. There are several possible explanations for this pattern. First, our synthetic

468 trait axes may have captured fundamental differences in the strategies of species for

469 tolerating climate-related stress, but not for exploiting local habitat heterogeneity. One of

470 the most important stresses in the bromeliad system is hydrologic variability, including

471 periods of drought. Some species are able to withstand drought with drought-resistant

472 eggs (e.g. Wyeomyia spp.: Dézerald et al. 2015), whereas others have drought-tolerant

473 larvae (e.g. Tipulidae larvae: Amundrud and Srivastava 2015). Many mosquito larvae are

474 sensitive to drought because their legless larvae require water to move (e.g. Culex spp.:

475 Amundrud and Srivastava 2015). However, odonates - a dominant predator in the food

476 web - are vulnerable to drought because of their long larval stage (Guzman et al. 2019,

477 Srivastava et al. 2020a). Therefore, multiple trait axes, as used in our study, are needed

478 to capture traits relevant to drought tolerance, including resistant life forms, larval

479 duration (i.e. cohort production interval), and pelagic requirements. Geographic patterns

480 in drought predict the distribution of invertebrate families that comprise the species pool 
481 of bromeliad invertebrates (Srivastava et al. 2020b) and families often have unique

482 functional traits (Céréghino et al. 2018). Climate is likely a better predictor of such

483 geographic patterns in drought prevalence than bromeliad water volume, as water

484 volume is only measured on the particular day of sampling and is very dynamic between

485 days. Invertebrate mortality rarely follows a single day of drought; instead mortality

486 ranges from 11-73\% after 18 days without water (Amundrud and Srivastava 2015). Similarly,

487 experimental exclusion of rainfall from French Guiana bromeliads led to changes in

488 functional trait composition of invertebrates only after six weeks without rain (Dézerald et

489 al. 2015). Although no single climate variable dominated the effects on traits, many of the

490 top climate variables were related to variation (daily or seasonal) in temperature and

491 precipitation as might be anticipated if climate affected traits via drought prevalence.

492 Our conclusion that temperature seasonality was an important determinant of trait

493 composition is similar to Swenson et al. (2012).

494 A second explanation is that deterministic filtering by local conditions is largely

495 overwhelmed by stochasticity in the colonization and emergence rates of invertebrates

496 from bromeliads. The majority of invertebrates in bromeliads are insects and thus have

497 complex life cycles, meaning that only the egg to larval or pupal stages are aquatic.

498 Larval development can be as fast as two weeks for mosquitoes, and the majority of

499 insects (except odonates) have cohort production intervals of less than 30 days (Dézerald

500 et al. 2017). This is a relatively short period for the amount of detritus, water or light to

501 limit their abundances, and suggests that abundances may be more affected by 
502 oviposition and predation - both of which have an important stochastic component.

503 Furthermore, because low abundances of species in bromeliads can indicate either

504 insufficient oviposition, successful completion of the larval stage and emergence, or

505 larval mortality, even deterministic effects of local factors may result in a complex array of

506 positive and negative effects on abundance. Given that the population dynamics of

507 species with complex life cycles (i.e. insects) occurs at scales larger than the bromeliad

508 (LeCraw et al. 2014), we might expect stronger trait-environment correlations to be found

509 at these larger scales, scales which are based on the bioclimatic zone and /or the

510 species pool.

511 A third possibility is that the suite of synthetic trait axes and local variables we used for

512 analysis somehow pre-determined greater matching of traits with climatic variables than

513 with local conditions. However, both the traits and local conditions used in this study

514 have been identified in previous studies as important factors determining community

515 composition (Richardson 1999, Usseglio-Polatera et al. 2000, Dézerald et al. 2014). The

516 four synthetic trait axes represent major fundamental niche dimensions such as trophic

517 position, habitat, life history and defence (Céréghino et al. 2018), and explained 45\% of

518 the total variance in species traits from the 12 traits we assembled. Although the main

519 goal of this study was to explain variations in those four main ecological strategies or four

520 PCA axes, other important ecological strategies (PCA5, PCA6, PCA7, . . ) could also be

521 influenced by local and bioclimatic conditions, however, these other axes were not

522 significant in (Céréghino et al. 2018)) and did not have biological interpretations. The four 
523 ecological strategies we studied here, have previously been identified as basic niche

524 dimensions in other systems and in other clades (Winemiller et al. 2015), suggesting that

525 they may be general across different types of communities, and perhaps, broadly

526 applicable to aquatic invertebrates in other ecosystems. Extensive research on bromeliad

527 communities has demonstrated that local conditions such as water volume, detrital

528 amount and canopy cover affects bromeliad community structure, including

529 predator:prey ratios and species richness in bromeliads (Richardson et al. 2000,

530 Srivastava et al. 2008, Dézerald et al. 2014). There are well-known mechanistic reasons

531 behind these relationships. The amount of light available to a bromeliad (i.e. canopy

532 cover) determines algal productivity, and therefore, the relative importance of detritus

533 versus algae in the diet of different macroinvertebrates (Farjalla et al. 2016). In general,

534 detritus is the main source of nutrients in the bromeliad food web, and its quantity is

535 related to overall macroinvertebrate biomass (Richardson et al. 2000). The amount of

536 water found in a bromeliad at the time of sampling is related to the amount of habitat

537 available to invertebrates, the risk of drought, and whether it is colonized by predators,

538 and as such, habitat size is an important predictor of species richness, species

539 composition and trophic structure (Srivastava et al. 2008, Amundrud and Srivastava

540 2015, Petermann et al. 2015). In an experiment where many of these factors were

541 controlled for, local variation in rainfall impacted the community structure of bromeliad

542 macroinvertebrates (Srivastava 2020b), and in extreme cases, caused an inversion of the

543 trophic pyramid (Romero et al. 2020). So a combination of local conditions will have

544 some effect on community dynamics and the distribution of traits. 
545 Our conclusion that climate overwhelms local conditions in driving community trait

546 structure contrasts to studies on plant communities by Bruelheide et al. (2018) who found

547 that micro-environmental gradients were more influential than climate. This may be

548 because two of the three local scale factors we analyzed varied more at biogeographical

549 than local scales (Table A2).

550 An important conclusion of our analysis is that there are cross-scale interactions between

551 environmental drivers of trait composition. That is, the effect of local conditions depends

552 on the regional climate. Specifically, the effect of either detrital amount or water volume

553 depended on temporal variation in precipitation and temperature at the field site. This

554 may reflect the ability of large detrital-filled bromeliads to buffer the effects of climate

555 variation on drought prevalence (Srivastava et al. 2020a). Studies of functional traits that

556 use coarse-grained data such as range maps or remote sensing data cannot test for such

557 cross-scale effects of the response of the community to local and climatic conditions.

558 However, there is a growing number of fine-grained datasets with a complete census of

559 the community for which interactions between local conditions and larger scale

560 environmental constraints can be tested. Such datasets are particularly well represented

561 by plants (e.g. Blonder 2018, Bruelheide et al. 2018), but also freshwater invertebrates

562 (e.g. Aspin et al. 2019), fish (e.g. Winemiller et al. 2015), intertidal organisms (e.g. Menge

563 et al. 1999) and marine coastal fishes (e.g. Hemingson and Bellwood 2018). The

564 challenge for analyzing cross-scale effects in these studies is not the large-scale climatic 
data, but rather the fine-grained environmental data that matters for resource acquisition, competition, predation and facilitation. Fine-grained microenvironmental data, only some of which was available in our study, is likely to be critical in determining the relative

568 importance of environmental filtering and biotic interactions as well as the degree of 569 context dependence (Blonder 2018). A particular advantage of our study was that we 570 were able to measure the entire aquatic macroinvertebrate community at a fine scale at 571 multiple locations across a wide biogeographic range, which then were assembled into a 572 large database, through the support of the French data synthesis centre, CESAB. The 573 randomized sub-sampling procedure was used to control for uneven sampling effort 574 between sites, and gave robust estimates of variance explained between sites. Although 575 sub-sampling reduces statistical power, we gained confidence in our among site 576 comparisons.

577 Overall, we found that climate explained more variation than local conditions, and that 578 the two scales interactively determine the functional traits of bromeliad

579 macroinvertebrate communities across their Neotropical range. Our ability to contrast the 580 effects of climatic vs. local conditions hinged on the compilation of multiple fine-grained 581 datasets. We argue that in addition to sampling communities at local scales, ecologists 582 should aggregate studies that span large ranges in climate variation in order to fully 583 understand trait filtering at local, regional and global scales.

\section{References}


Amundrud, S. L. and Srivastava, D. S. 2015. Drought sensitivity predicts habitat size sensitivity in an aquatic ecosystem. - Ecology 96: 1957-1965.

Amundrud, S. L. et al. 2018. Dispersal barriers and climate determine the geographic distribution of the helicopter damselfly Mecistogaster modesta. - Freshwater Biology 63: $214-223$.

Anderson, M. J. 2001. A new method for non-parametric multivariate analysis of variance. - Austral Ecology 26: 32-46.

Aspin, T. W. H. et al. 2019. Extreme drought pushes stream invertebrate communities over functional thresholds. - Glob. Chang. Biol. 25: 230-244.

Bates, D. et al. 2015. Fitting linear mixed-effects models using Ime4. - J. Stat. Softw. 67: $1-48$.

Blonder, B. 2018. Hypervolume concepts in niche- and trait-based ecology. - Ecography 41: 1441-1455.

Bruelheide, H. et al. 2018. Global trait-environment relationships of plant communities. Nat Ecol Evol 2: 1906-1917.

Céréghino, R. et al. 2011. Ant-plant mutualisms promote functional diversity in phytotelm communities. - Functional Ecology 25: 954-963.

Céréghino, R. et al. 2018. Constraints on the functional trait space of aquatic invertebrates in bromeliads. - Functional Ecology 32: 2435-2447.

Chase, J. M. and Leibold, M. A. 2004. Ecological niches: linking classical and contemporary approaches. - Biodivers. Conserv. 13: 1791-1793.

Chave, J. 2013. The problem of pattern and scale in ecology: what have we learned in 20 years? - Ecol. Lett. 16: 4-16.

Chave, J. et al. 2009. Towards a worldwide wood economics spectrum. - Ecol. Lett. 12: 351-366.

Chesson, P. 2000. Mechanisms of maintenance of species diversity. - Annu. Rev. Ecol. Syst. 31: 343-366.

Damos, P. and Savopoulou-Soultani, M. 2012. Temperature-driven models for insect development and vital thermal requirements. - Psyche: A Journal of Entomology 2012: 1-13.

de Omena, P. M. et al. 2019. Consumptive effects and mismatch in predator-prey turnover rates cause inversion of biomass pyramids. - Oecologia 190: 159-168. 
648 Hubbell, S. P. 2011. The unified neutral theory of biodiversity and biogeography (MPB-32).

Dézerald, O. et al. 2014. Environmental determinants of macroinvertebrate diversity in small water bodies: insights from tank-bromeliads. - Hydrobiologia 723: 77-86.

Dézerald, O. et al. 2015. Functional trait responses of aquatic macroinvertebrates to simulated drought in a Neotropical bromeliad ecosystem. - Freshwater Biology 60: 1917-1929.

Dézerald, O. et al. 2017. Environmental drivers of invertebrate population dynamics in Neotropical tank bromeliads. - Freshwater Biology 62: 229-242.

Dézerald, O. et al. 2018. Functional traits and environmental conditions predict community isotopic niches and energy pathways across spatial scales. - Functional Ecology 32: 2423-2434.

Diamond, J. M. 1975. The island dilemma: lessons of modern biogeographic studies for the design of natural reserves. - Biol. Conserv. 7: 129-146.

Estes, J. A. et al. 2011. Trophic downgrading of planet Earth. - Science 333: 301-306.

Farjalla, V. F. et al. 2016. Terrestrial support of aquatic food webs depends on light inputs: a geographically-replicated test using tank bromeliads. - Ecology 97: 2147-2156.

Fick, S. E. and Hijmans, R. J. 2017. WorldClim 2: new 1-km spatial resolution climate surfaces for global land areas. - Int. J. Climatol. 37: 4302-4315.

Garnier, E. et al. 2004. Plant functional markers capture ecosystem properties during secondary succession. - Ecology 85: 2630-2637.

Guzman, L. M. et al. 2019. Towards a multi-trophic extension of metacommunity ecology. - Ecology Letters 22: 19-33.

Hammill, E. et al. 2015. Behavioural responses to predation may explain shifts in community structure. - Freshwater Biology 60: 125-135.

Hanski, I. 1999. Habitat connectivity, habitat continuity, and metapopulations in dynamic landscapes. - Oikos 87: 209-219.

Hawkins, B. A. et al. 2017. Structural bias in aggregated species-level variables driven by repeated species co-occurrences: a pervasive problem in community and assemblage data. - Journal of Biogeography 44: 1199-1211.

Hemingson, C. R. and Bellwood, D. R. 2018. Biogeographic patterns in major marine realms: function not taxonomy unites fish assemblages in reef, seagrass and mangrove systems. - Ecography 41: 174-182. 
- Princeton University Press.

Kraft, N. J. B. and Ackerly, D. D. 2010. Functional trait and phylogenetic tests of community assembly across spatial scales in an Amazonian forest. - Ecological Monographs 80: 401-422.

653 Kratina, P. et al. 2017. Environmental control of the microfaunal community structure in 654 tropical bromeliads. - Ecol. Evol. 7: 1627-1634.

655 Lamanna, C. et al. 2014. Functional trait space and the latitudinal diversity gradient. $656 \quad$ Proc. Natl. Acad. Sci. U. S. A. 111: 13745-13750.

657 Lande, R. et al. 2003. Stochastic population dynamics in ecology and conservation. 658 Oxford University Press.

659 LeCraw, R. M. et al. 2014. Metacommunity size influences aquatic community composition 660 in a natural mesocosm landscape. - Oikos 123: 903-911.

661 Legendre, P. and Gallagher, E. D. 2001. Ecologically meaningful transformations for 662 ordination of species data. - Oecologia 129: 271-280.

663 Levin, S. A. 1992. The problem of pattern and scale in ecology: the Robert H. MacArthur 664 Award Lecture. - Ecology 73: 1943-1967.

665 Loreau, M. et al. 2001. Biodiversity and ecosystem functioning: current knowledge and 666 future challenges. - Science 294: 804-808.

667 MacArthur, R. H. and Wilson, E. O. 1967. The theory of island biogeography. - Princeton $668 \quad$ University Press.

669 McGill, B. J. et al. 2006. Rebuilding community ecology from functional traits. - Trends $670 \quad$ Ecol. Evol. 21: 178-185.

671 Menge, B. A. et al. 1999. Top-down and bottom-up regulation of New Zealand rocky 672 intertidal communities. - Ecological Monographs 69: 297.

673 Merritt, R. W. and Cummins, K. W. 1996. An Introduction to the Aquatic Insects of North 674 America. - Kendall Hunt.

675 Moore, J. C. et al. 2004. Detritus, trophic dynamics and biodiversity. - Ecology Letters 7: 676 584-600.

677 Oertli, B. et al. 2002. Does size matter? the relationship between pond area and 678 biodiversity. - Biological Conservation 104: 59-70.

679 Oksanen, J. et al. 2017. Vegan: Community Ecology Package.

680 Peres-Neto, P. R. and Kembel, S. W. 2015. Phylogenetic gradient analysis: environmental 
712 Srivastava, D. S. et al. 2020b. Ecological response to altered rainfall differs across the drivers of phylogenetic variation across ecological communities. - Plant Ecology 216: 709-724.

Petermann, J. S. et al. 2015. Dominant predators mediate the impact of habitat size on trophic structure in bromeliad invertebrate communities. - Ecology 96: 428-439.

Pianka, E. R. et al. 2017. Toward a periodic table of niches, or exploring the lizard niche hypervolume. - Am. Nat. 190: 601-616.

R Core Team 2016. R: A Language and Environment for Statistical Computing. R Foundation for Statistical Computing, Vienna, Austria.

Richardson, B. A. 1999. The bromeliad microcosm and the assessment of faunal diversity in a neotropical forest. - Biotropica 31: 321-336.

Richardson, B. A. et al. 2000. Effects of nutrient availability and other elevational changes on bromeliad populations and their invertebrate communities in a humid tropical forest in Puerto Rico. - Journal of Tropical Ecology 16: 167-188.

Ricklefs, R. E. 2012. Species richness and morphological diversity of passerine birds. Proc. Natl. Acad. Sci. U. S. A. 109: 14482-14487.

Ricklefs, R. E. and Schluter, D. 1993. Species diversity in ecological communities: historical and geographical perspectives. - University of Chicago Press.

Romero, G. Q. et al. 2018. Global predation pressure redistribution under future climate change. - Nature Climate Change 8: 1087-1091.

Romero, G. Q. et al. 2020. Extreme rainfall events alter the trophic structure in bromeliad tanks across the Neotropics. - Nat. Commun. 11: 3215.

Schmitz, O. J. et al. 2015. Functional traits and trait-mediated interactions: connecting community-level interactions with ecosystem functioning. - In: Pawar, S. et al. (eds), Trait-based ecology - from structure to function. Advances in ecological research. Academic Press, pp. 319-343.

Srivastava, D. S. and Bell, T. 2009. Reducing horizontal and vertical diversity in a foodweb triggers extinctions and impacts functions. - Ecology Letters 12: 1016-1028.

Srivastava, D. S. et al. 2008. Why are predators more sensitive to habitat size than their prey? insights from bromeliad insect food webs. - Am. Nat. 172: 761-771.

Srivastava, D. S. et al. 2020a. Habitat size thresholds for predators: why damselflies only occur in large bromeliads. - Biotropica in press. 
$713 \quad$ Neotropics. - Ecology in press.

714 Swenson, N. G. 2014. Functional and phylogenetic ecology in R. - Use R! in press.

715 Swenson, N. G. et al. 2012. The biogeography and filtering of woody plant functional

716 diversity in North and South America. - Global Ecology and Biogeography 21:

717 798-808.

718 Usseglio-Polatera, P. et al. 2000. Biological and ecological traits of benthic freshwater

719 macroinvertebrates: relationships and definition of groups with similar traits. -

$720 \quad$ Freshwater Biology 43: 175-205.

721 Vellend, M. 2016. The theory of ecological communities (MPB-57). - Princeton University

722 Press.

723 Violle, C. et al. 2014. The emergence and promise of functional biogeography. - Proc.

724 Natl. Acad. Sci. U. S. A. 111: 13690-13696.

725 Winemiller, K. O. et al. 2015. Functional traits, convergent evolution, and periodic tables of 726 niches. - Ecol. Lett. 18: 737-751.

727 Zelený, D. 2018. Which results of the standard test for community-weighted mean 728 approach are too optimistic? - Journal of Vegetation Science 29: 953-966. 